\title{
EMBEDDED SEMICONDUCTOR QUANTUM DOTS - CURRENT OVERVIEW
}

\author{
Eduard HULICIUS, Alice HOSPODKOVÁ, Markéta ZÍKOVÁ \\ Department of Semiconductors, Institute of Physics, Czech Academy of Sciences, v.v.i., Prague, \\ Czech Republic,EU, hulicius@fzu.cz
}

https://doi.org/10.37904/nanocon.2020.3680

\begin{abstract}
This review paper focuses on semiconductor quantum dots (QDs) embedded inside semiconductor heterostructures prepared by Metalorganic Vapor Phase Epitaxy (MOVPE) technology and based on our contribution in [1]. Semiconductor direct-bandgap materials have much higher energy conversion efficiency than the light emission from atoms/molecules in a glass matrix or from most other light sources, but they have a broad band or multimode light emission spectra. QDs created and embedded inside semiconductor heterostructures can fundamentally improve the quality of emitted light spectrum, temperature dependencies and efficiency of emission.

QDs exhibit unique electronic and optical properties, intermediate between those of bulk semiconductors and discrete atoms or molecules. The reason for this is that the size of QDs is comparable with the de Broglie wavelength of an electron in a crystal. Electrons (and holes) inside QDs behave differently from that found in a "bulk" material. The most important difference is that inside the QDs, electrons (and holes) can occupy only discrete energy levels due to strong localization. The position of energy levels depends mainly on the smallest dimension of a QD. Applications include QD lasers for integrated silicon photonics and quantum computing, QD LEDs for highly efficient solid-state lighting, QDs for intermediate band solar cells and multi-exciton generation and QDs for bio-medical labeling, imaging, targeted drug delivery, sensing, and therapy.
\end{abstract}

Keywords: Semiconductors, quantum dots, quantum levels, epitaxy, applications

\section{INTRODUCTION}

This contribution focuses only on quantum dots (QDs) embedded inside a semiconductor structure. Freestanding QDs (semiconductor, colloidal, metal etc.) with a much larger application field, which can be prepared from the same material, are not subject of this talk. A research report about the whole family of QDs market can be found at (paid) [2].

QDs are small pieces of semiconductor nanocrystals (mainly) with sizes in the range from ones to tens of nanometers. Ekimov and Onushchenko published the first idea of (freestanding) QDs, having properties different from that of the bulk material, in 1981 [3]. During the early 1990s, embedded QDs were prepared by molecular beam epitaxy (MBE) [4-5] in several laboratories, as described in [6].

Light emission (LED or laser type) from separated atoms in a glass matrix, e.g. neodymium doped YAG, ruby, etc., or from atoms or molecules in a gas, is nearly monochromatic thanks to these media having sharp energy levels.

Semiconductor direct-bandgap materials have much higher energy conversion efficiency than indirectbandgap ones, but many possible recombination energies, thus they have broadband or multimode light emission. Electron energy levels in isolated atoms, the bulk semiconductor material (direct) and inside a QD are shown in Figure 1. 


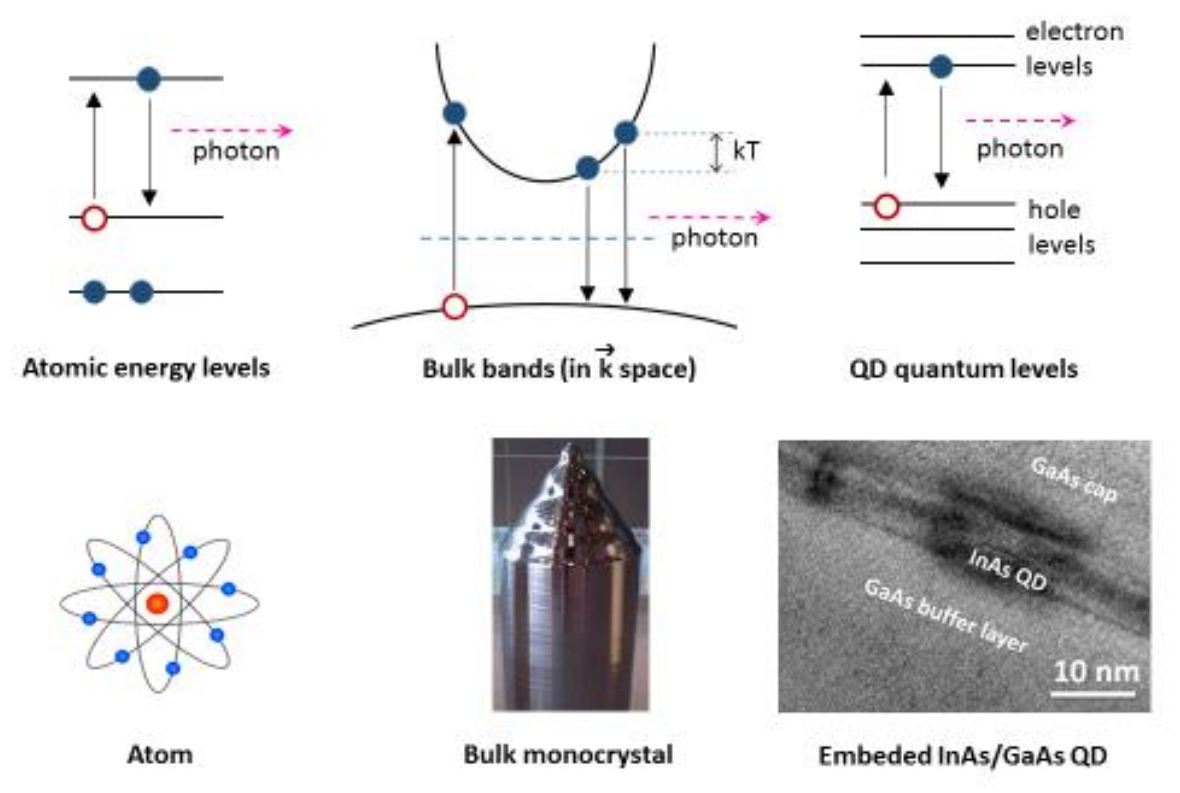

Figure 1 Paradigm of quantum dots, from [1].

A QD, resembling an artificial atom in its ability to confine the electron (or hole) wavefunction, emits more monochromatic light than the bulk material.

Reasons and examples of QD parameters for semiconductor lasers are as follows: The emission from quantum levels in QWs, QWrs or QDs is monochromatic and still very efficient. One of the effects of strong quantum confinement are discrete energy levels. Another effect is a lower threshold current density of a QD-based semiconductor laser, when achieving the inversion of population in QDs, which is needed for a stimulated emission, as well as suppression of loss mechanisms such as Auger recombination and intervalence band absorption.

The photon energy (emitted wavelength) can be changed mainly by the size of QDs and not only by chemical composition. This is a similar principle as in quantum well lasers, but the temperature dependence of these lasers is also much lower than for "classical" two-dimensional QW based lasers. Another very important property of QD-based semiconductor lasers is that very small QDs can emit single photons, which is necessary for quantum cryptography.

\section{2. $\quad A^{\prime \prime \prime} B^{V}$ MATERIALS AND STRUCTURES}

\subsection{QDs embedded in the structure}

The three main growth technological procedures used for MOVPE preparation of QDs embedded in the structure are: 1) self-assembled Stranski-Krastanov growth mode; 2) formation of QDs in prepatterned inverted pyramids and 3) droplet epitaxy. The most widely used procedure is a self-assembling of QDs in SK growth mode. This growth mode can be used for highly strained QDs such as InAs on GaAs, InSb on GaSb, or InGaAs on GaP. In this technological procedure, QDs form from the material of a strained epitaxial layer, when the system has enough time to find the minimum energy, this means either the growth of strained layer has to be slow or the growth is interrupted for a few seconds after the epitaxial growth of the strained layer. By this method, different QD densities can be obtained (108-1011 cm-2) depending on the amount of deposited strained material and the time of the growth interruption. It is possible to grow stacks of vertically aligned QDs when the separation layer thickness between QDs is sufficiently thin $(3-10 \mathrm{~nm})$. In such a case, QDs form on the top of the QDs in previous layer thanks to the advantageous lattice deformation. An atomic force 
microscopy (AFM) picture of the surface of a vertically correlated QD structure with the last uncovered QD layer is shown in Figure 2a). It nicely demonstrates that the vertical correlation of QDs is high. No surface QDs form between hillocks, which are formed above buried QDs in previous layers and the QDs are formed on those hillocks only. A transmission electron microscopy (TEM) example of the cut of a similar structure is shown in Figure 2b).

a)

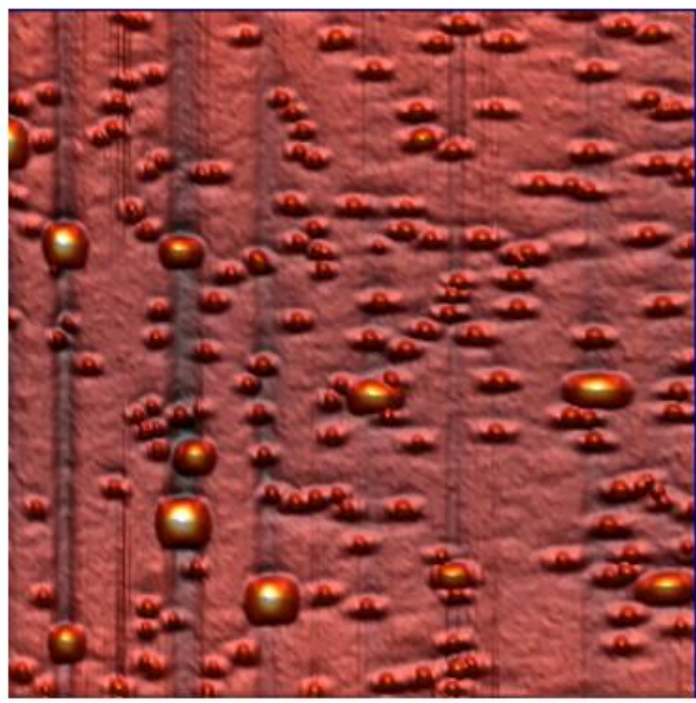

b)

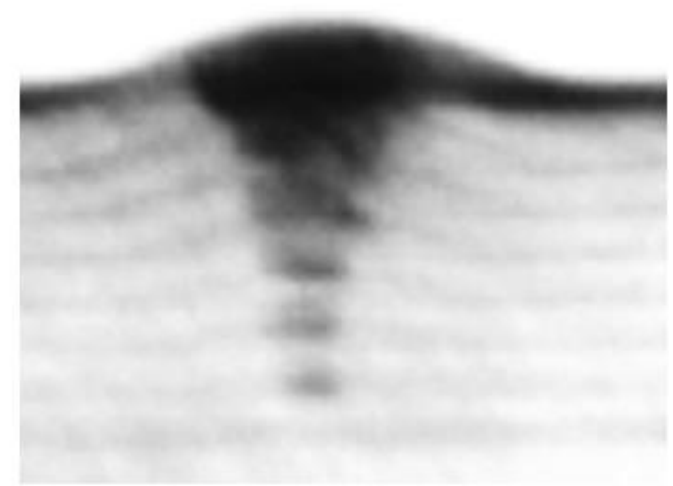

Figure 2 AFM picture of a surface with vertically correlated QDs with the last uncovered QD layer (a); TEM picture of the cut of a 7-layer vertical QD structure (b). Taken from [1].

\subsection{Strain reducing and capping layers}

There are two commonly used ternary materials for the growth of strain-reducing layers from the V-III group semiconductors, InGaAs and GaAsSb that have been studied simultaneously during recent years. The mechanism of the strain relaxation is the same for both InGaAs and GaAsSb ternary alloys, but at the same time different from the GaAs-covering mechanism. While GaAs is covering the surface with QDs from the bottom upwards and is leveling off the surface, both InGaAs and GaAsSb layers copy the topography of the surface, see Figure 3, which then enables the preservation of the size and shape of InAs QDs.
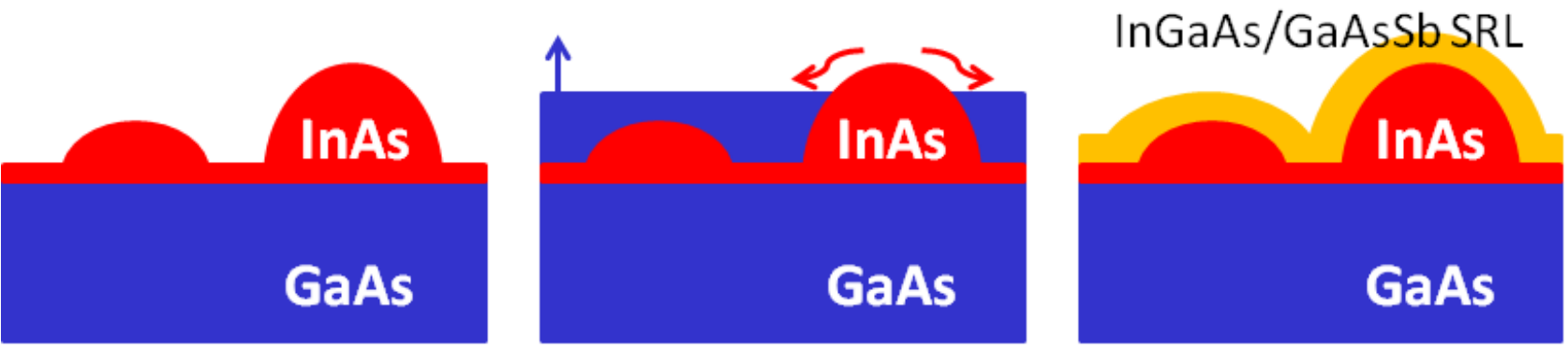

Figure 3 Covering mechanism of different types of covering layers from left: no capping layer, GaAs capping layer, InGaAs/GaAsSb strain-reducing layer. Taken from [1].

For InGaAs SRL, a similar study of the capping-layer thickness, as was carried out for the GaAs CL, was performed. The thickness of the InGaAs was kept at $5 \mathrm{~nm}$, while the thickness of the GaAs was varied. The results for InGaAs with $13 \%$ of In are shown in Figure 4. When InGaAs strain-reducing layers are used, it is possible to achieve lower recombination energy. The main problem of the InGaAs SRL is in the weak confinement of electrons in the QD for higher In content due to the lowering of the conduction-band edges, which can then lead to a higher dependency on the ambient conditions such as temperature. Another reason 


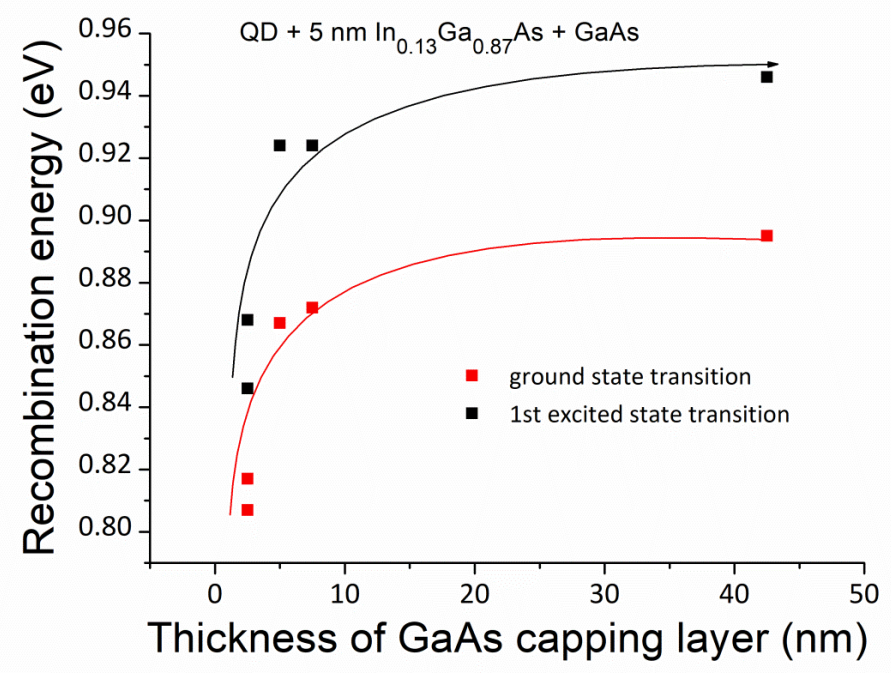

Figure 4 Dependence of recombination energy of a structure with InGaAs strain-reducing layer on the thickness of GaAs capping layer. Taken from [1].

for the weak confinement could be also a poor InAs/InGaAs interface that could lead to delocalization of electrons in the structure. Then, the structure shows photoluminescence (PL) with low intensity because the recombination rate of electrons and holes decreases. This can be seen in Figure 5 from PL spectra measured for different InGaAs composition. There, the PL energy is decreased for higher In content, but from $13 \%$ of In the PL intensity is steadily decreasing. Concerning the PL maximum shift and intensity, the most suitable composition of the InGaAs layer would be around $13 \%$ or slightly higher.

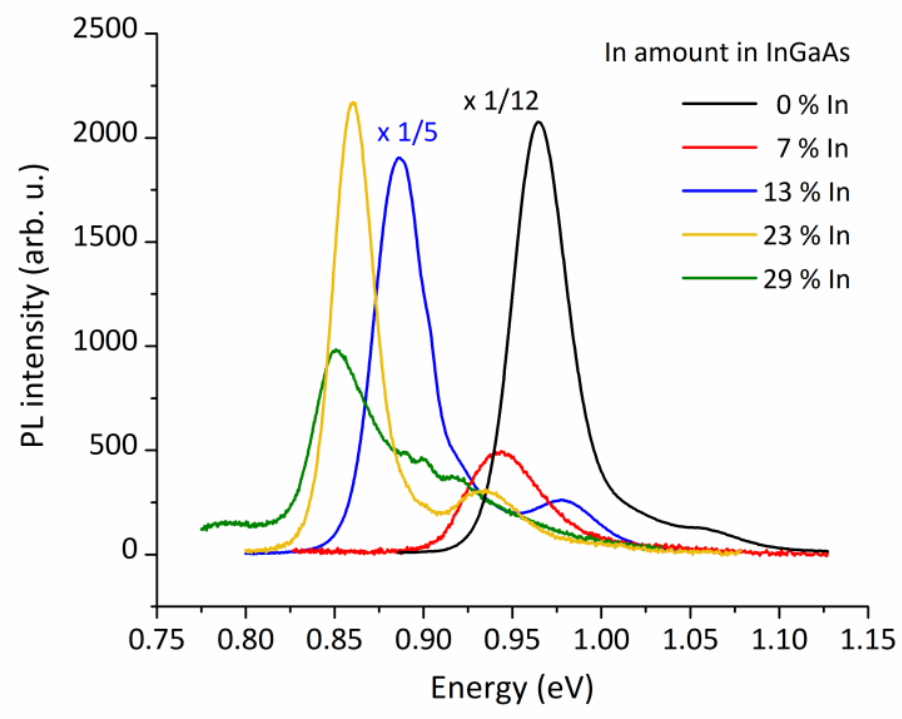

Figure 5 PL spectra of InAs QDs covered by InGaAs SRL with different In content. Taken from [1].

\section{REFLECTANCE ANISOTROPY SPECTROSCOPY FOR QD GROWTH CONTROLLING}

Compared to conventional reflectance measurements, Reflectance Anisotropy Spectroscopy (RAS) monitors in situ the properties of surfaces, interfaces and doping-induced surface electric fields, which makes it a unique optical in-situ technique. Moreover, because RAS includes the conventional reflectance measurement in addition to its surface and doping level sensitivity, the method allows for determination of layer thickness, 
growth rate and composition through reflectance analysis. Note that RAS can only be applied to cubic crystals, as they are grown e.g. on GaAs and InP. For bulk material of cubic crystals, the reflectance should be the same along two axes perpendicular to each other. However, at the surface region symmetry is reduced due to the crystal termination. This region of the crystal is responsible for the occurrence of the RAS signal. It carries information about symmetry and stoichiometry of the uppermost atomic layers. As tiny changes in surface stoichiometry can have a considerable effect on the surface symmetry, RAS enables growth monitoring and growth control - see Figure 6, even on a sub-monolayer level - see Figure 7.
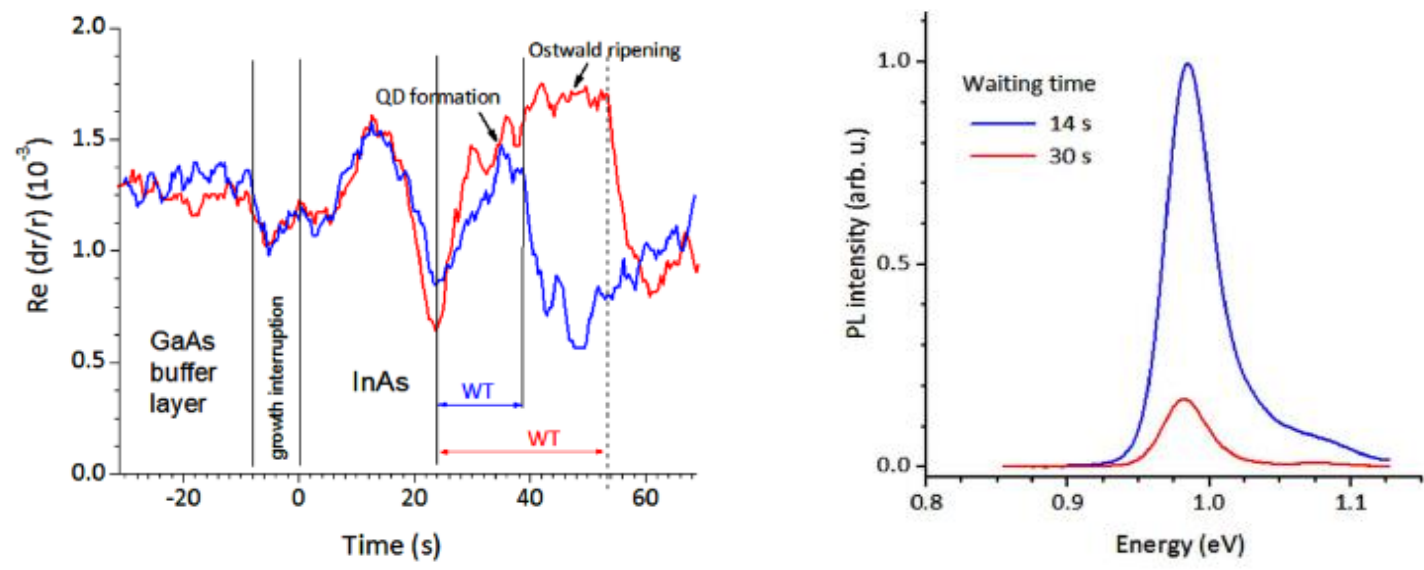

Figure 6 Left: RAS spectra (for $2.65 \mathrm{eV}$ ) of samples with different waiting times (WT) - time during which QDs are formed - for $14 \mathrm{~s}$ WT (blue line) QD were formed, for $\mathbf{3 0} \mathrm{s}$ WT (red line) the time was overly long and QD coalesced into large objects. (This process is called Ostwald ripening.). Right: QDs were formed and evince PL for a sample with $14 \mathrm{~s}$ waiting time (WT), (blue line), for the WT of $30 \mathrm{~s}$ (red line). QDs coalesced into large objects that do not have a PL. Taken from [7].
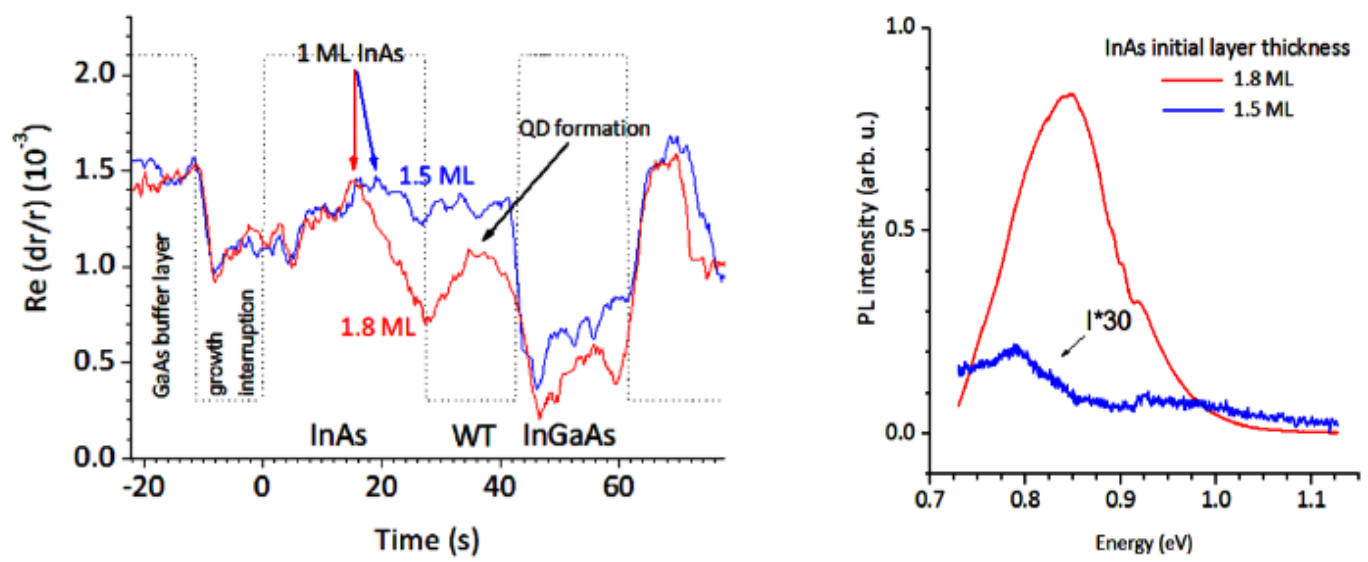

Figure 7_Left: RAS spectrum (for $2.65 \mathrm{eV}$ ) for estimation of the proper amount of InAs for a QD formation for 1.8 ML thick InAs layer (red line), 1.5 ML InAs (blue line). Right: PL spectra of samples with 1.8 ML and 1.5 ML of InAs for a QD formation, only inside the sample with 1.8 ML QDs were created and contribute to PL. Taken from [7].

\section{CONCLUSION}

This paper explained importance of QDs embedded in semiconductor heterostructures prepared by MOVPE). It shows some technology parameters important for QD properties and explains the basic in-situ characterization technique RAS, which can be used for controlling of QD growth. 
Applications include QD lasers for integrated silicon photonics and quantum computing. QD LEDs for highly efficient solid-state lighting. QDs for intermediate band solar cells and multi-exciton generation. QDs for biomedical labeling, imaging, targeted drug delivery, sensing, and therapy.

\section{Future applications:}

- QD-based single-photon or -electron devices. Mainly single-photon QD lasers for quantum cryptography.

- $\quad$ QD MIR photomultipliers can be more useful than other QD photomultiplier wavelengths applications.

- $\quad$ QD long-life memories are very promising.

- $\quad$ Telecom lasers very probably for $1.3 \mu \mathrm{m}$, maybe also for $1.55 \mu \mathrm{m}$.

- $\quad$ Prospects of QD solar cells: at a very early stage of research, complications to be expected.

\section{Future research:}

- $\quad$ Extension of QD device wavelengths to the far-MIR and to the UV using different materials as well as size and shape of QDs.

- Improvement of structure parameters over the standard ones.

- The cost of devices has to be reduced, as usually, thus research of low-cost and mature (Si or GaAs wafers based?) technology has to be improved.

\section{ACKNOWLEDGEMENTS}

The authors acknowledge the support of MSMT project no. NPU LO1603 - ASTRANIT.

\section{REFERENCES}

[1] E. HULICIUS, A. HOSPODKOVÁ, M. ZíKOVÁ. Quantum dots. In: IRVINE, S.J.C., CAPPER, P., eds, Metalorganic Vapor Phase Epitaxy (MOVPE): Growth, Materials Properties and Applications. John Wiley \& Sons, Hoboken. 2020, Chapter 6, p. 584.

[2] Press release from: WiseGuyResearch Consultants Pvt Ltd_Project Portfolio Quantum Dots 2017 Global Market Expected to Grow at CAGR 11.39\% and Forecast to 2019, Quantum Dots Market Research Report 2017.

[3] EKIMOV, A.I. and ONUSHCHENKO, A.A. Quantum size effect in three-dimensional microscopic semiconductor crystals. JETP Letters. 1981, vol. 34, pp. 345-349.

[4] LEONARD, 2 ., KRISHNAMURTHY, M., REAVES, C.M., et al. Direct formation of quantum-sized dots from uniform coherent islands of InGaAs on GaAs surfaces. Applied Physics Letters. 1993, vol. 63, pp. 3203-3205.

[5] GRUNDMANN, M., CHRISTEN, J., LEDENTSOV, N.N., et al. (1995) Ultranarrow Luminescence Lines from Single Quantum Dots. Physical Review Letters. 1995, vol. 74, pp. 4043-4046.

[6] PETROFF, P.M. and DENBAARS, S.P. MBE and MOCVD growth and properties of self-assembling quantum dot arrays in III-V semiconductor structures. Superlattices and Microstructures. 1994, vol. 15, pp. 15-21.

[7] ZíKOVÁ, M., HOSPODKOVÁ, A. PANGRÁC, J., VYSKOČIL, J., HULICIUS, E., OSWALD, J., KOMNINOU, P, KIOSEOGLOU, J. The Metalorganic Vapour Phase Epitaxy Growthof AlllBVHeterostructuresObserved by Reflection Anisotropy Spectroscopy. In: Proceedings the 44th International School and Conference on the Physics of Semiconductors "Jaszowiec 2015". Wisła 2015. 